\title{
El Análisis Funcional de Conducta en la Evaluación Psicológica Forense de la Credibilidad del Testimonio
}

Functional Analysis of Behavior in Forensic Psychological Evaluation of Statement Credibility

Ramón Jesús Vilalta Suárez ${ }^{1}$

Máximo Winberg Nodal ${ }^{2}$

(1) Instituto de Medicina Legal y Ciencias Forenses de Oviedo, Oviedo, España.

(2) Instituto de Medicina Legal y Ciencias Forenses de Toledo, Toledo, España.

Email correspondencia: rvilalta@cop.es

\section{Resumen}

Se pretende describir un método de evaluación psicológica de la credibilidad del testimonio, que incluye diversas técnicas que aparentemente se utilizan por separado, añadiendo transversalmente el Análisis Funcional de Conducta (AFC). Se trata de un método aplicado a la práctica forense en formato de evaluación de caso único de medidas repetidas. Se describen seis componentes no estancos en la evaluación: el estudio del expediente, el planteamiento de la evaluación, la entrevista, la técnica CBCA-SVA, la detección del engaño y el Análisis Funcional de Conducta. Este último engloba toda la evaluación, pues da cuenta de la funcionalidad de las conductas, cogniciones y emociones del explorado: de la víctima genuina en el formato de Condicionamiento Clásico propio de su condición, y de las denuncias instrumentales a través del paradigma del Condicionamiento Operante. Esta perspectiva pretende incrementar la especificidad de la evaluación psicológica de la credibilidad del testimonio.

Palabras clave: Evaluación Psicológica, Credibilidad, Análisis Funcional de Conducta.

\section{Summary}

This paper describes a psychological evaluation method of statement credibility, which includes various techniques apparently used separately, adding a Functional Analysis of Behavior (ABC) transversely. This is an applied method of forensic psychological evaluation in a repeated measures design $(n=1)$. Six not hermetic components in the evaluation are described: the file study, the evaluation plan, the interview, the CBCA-SVA, the detection of deception and the Functional Analysis of Behavior. The latter covers the entire evaluation, as it explains the function of behaviors, cognitions and emotions of the person being explored: the genuine victim in the Classical Conditioning, and the instrumental allegations through Operanting Conditioning. This perspective enhances the specificity of the psychological evaluation of the credibility of the testimony.

Key words: Psychological evaluation, Credibility, Functional Analysis of Behavior. 
Vilalta, R. J., Winberg, M. (2016) El análisis funcional de conducta en la evaluación psicológica forense de la credibilidad del testimonio. Behavior \& Law Journal 2 (1), 1-10

\section{INTRODUCCIÓN}

La colaboración del psicólogo forense en el campo de la investigación forense y policial del testimonio de víctimas de delitos sexuales, viene avalada por la consideración del mismo como especialista en su análisis, sobre todo a partir del Análisis de la Credibilidad de las Declaraciones SVACBCA (Steller y Köhnken, 1989). Dicha evaluación psicológica, cobra especial relevancia cuando no se cuenta con otras pruebas sobre los hechos denunciados y suele incluir la valoración de las posibles consecuencias psicológicas del delito, algo también complicado, pues la vivencia de una situación traumática no produce necesariamente unas secuelas que el psicólogo pueda acreditar (Muñoz, 2013a).

El método que se propone para la valoración de la credibilidad en este artículo se describe de modo lineal, por pasos, pero comprendiendo que en la práctica cada caso seguirá su propio camino, haciendo innecesario recorrerlo por entero si ya se ha resuelto con alguna deducción previa, o yendo directamente al componente que lo puede resolver si así se revela en el planteamiento intelectual del mismo, que debe ser el hipotético-deductivo.

Los componentes que describimos aquí no están compartimentalizados, se comunican. Forman un círculo que se empieza por donde se debe en atención al caso y en el que, a través de sus radios, el psicólogo puede poner en relación cualquier punto en Análisis Funcional (AFC) para encontrar la deducción correcta. Aderezaremos la descripción de nuestro método de evaluación psicológica con ejemplos obtenidos de la experiencia forense:

Una chica de 13 años denunciaba una agresión sexual por parte de su padrastro, que se habia descubierto por unas anotaciones suyas en su diario. La exploración ginecológica descartaba daño alguno. Llamó la atención que la madre asegurase en su declaración que no tenía conocimiento de la existencia del diario; sin embargo al inicio del mismo constaba una dedicatoria suya. ¿Tenía alguna función que manifestase desconocer su existencia? De no haber sido por el diario nadie lo hubiera descubierto, y alegar tal desconocimiento podría ser funcional si se pretendía que el diario fuera un señuelo para dotar de credibilidad a una denuncia fabricada.
Los padres de la SVA-CBCA ya señalaban que su propuesta se trataba de una aproximación psicodiagnóstica (Steller y Köhnken, 1989) y parecían alejarse de la propuesta seminal de Trankell (1972) que ellos consideraban holístico-intuitiva (Manzanero, 2001), pero que resultaba ser claramente hipotéticodeductiva (Manzanero y González, 2015), pues además de mediante criterios de calidadreiteradamente atiende al control del testimonio a través de la lógica formal y del conocimiento empírico. Esta es nuestra inspiración en una tarea eminentemente práctica, aplicada a la realidad de la investigación criminal. Evidentemente, la lógica está por encima de los criterios de calidad narrativa de un testimonio cuando éste se presenta preparado o en circunstancias irreales; y no es intuitiva (Scott y Manzanero, 2015), sino fruto del trabajo intelectual sistemático.

La madre de una niña de 10 años, denunciaba unos abusos sexuales a la menor realizados por un tío político. La menor ya habia sido diagnosticada de un Sindrome de Estrés Postraumático y luego había sido ingresada varios días con un cuadro de amnesia postraumática. Ante la policía habia narrado un episodio en el que el supuesto agresor le ordenaba una felación, que ella invirtiendo el rol propio de una víctima- desdeñaba con éxito, recurriendo el agresor a desplazar sus lúbricos instintos a un gato que por alli merodeaba, acercando en cuenta su pene a las fauces del felino. Pensemos en dientes y lengua gatuna, para advertir cómo lo bubiera podido lamentar, detalle ausente del relato infantil. Aqui la deducción está en la propia naturaleza de lo narrado, sometido a lógica formal y conocimiento empírico. El AFC revelaba la ganancia secundaria de la denuncia para una familiar de la madre, que además era una persona con gran necesidad de atención y una bija muy sugestionable.

Proponemos un análisis hipotético-deductivo, de caso único, con lápiz y papel, empleando todos los conocimientos y técnicas de la evaluación psicológica. Describimos las herramientas, pero cada psicólogo debe saber la que utiliza, porqué y en qué orden, para explicar la pieza de conducta concreta que resuelve la investigación sobre la credibilidad de un testimonio: empleando la deducción, sujeta a comprobación y refutable por el mismo método. Como expresa Muñoz (2013b), en el ámbito forense se trata de realizar un 
Vilalta, R. J., Winberg, M. (2016) El análisis funcional de conducta en la evaluación psicológica forense de la credibilidad del testimonio. Behavior \& Law Journal 2 (1), 1-10

análisis global e integral de la realidad psicológica de la persona, más allá del simple uso nosológico de las etiquetas diagnósticas. Esto lo consigue el AFC.

\section{PRIMER COMPONENTE: ESTUDIO DEL EXPEDIENTE}

Primer paso obligado en la preparación de una investigación psicológica forense (Manzanero y González, 2015). Se trata de una tarea intelectual en la que puede emplearse un enfoque analítico puro, procurando un análisis contextual que mejore nuestra capacidad de detectar verdades y mentiras (Masip y Herrero, 2015a) indicios claves que “...permiten detectar mentiras en el mundo real' (Masip y Herrero, 2015b). Toda insistencia en su importancia resulta escasa, pues son numerosos los informes de credibilidad que quedan solventados con este trámite.

El Análisis de Validez (SVA) impone sin duda un buen conocimiento de todas las declaraciones previas y del contexto de revelación del abuso, pero tener en mente la perspectiva del AFC aún obliga a mayor detalle. El expediente revela al psicólogo bien dispuesto datos para el análisis topográfico de las conductas del denunciante, de la víctima o de otros implicados, y también si desvelan algún tipo de función, entendiendo que padecer un delito grave no resulta -casi nunca- funcional y que es muy posible encontrar el origen contaminado de la denuncia infundada o la función psicológica de la directamente falsa: su motivación.

El Análisis Funcional abre un ámbito de trabajo propio de la Psicología, pues permite el diagnóstico diferencial de cada asunto en un formato de diseño experimental de caso único de medidas repetidas $\mathrm{AB} A B$, en el que encaja toda la evaluación psicológica del sujeto y su testimonio, lo que aumenta la calidad de la evaluación, pues se constituye en un modelo que compendia reiterados indicadores de convergencia (Muñoz, 2013b).

La inspiración que ofrece a la credibilidad tal planteamiento deductivo, es que la víctima de un acontecimiento traumático debe responder a un paradigma de Condicionamiento Clásico, con presencia o no de estímulos condicionados al mismo. El individuo es ajeno a unas circunstancias que no controla, como el sujeto experimental del modelo pavloviano. Mostrará quizás algún tipo de condicionamiento ante la experiencia más o menos aversiva que denuncia. Tiene que ser posible explicar su evolución -mórbida o no- evaluando: su historia de vida y personalidad, las características del trauma y sus posibles cambios conductuales, cognitivos y emocionales.

Por contra la pseudovíctima, o es víctima de una sospecha infundada, o un sujeto operante cuya conducta puede describirse en términos de Condicionamiento Operante, pues participa activamente en la construcción artificiosa del engaño y lo va mostrando dentro de la propia dinámica de la evaluación (diagnosticable en los términos propios de un diseño de caso único de medidas repetidas $A B$ AB). Algo que también posibilita la actitud estratégica del psicólogo forense en un proceso dinámico de evaluación (Porter y Brinke, 2010; Masip y Herrero, 2015b).

Esta perspectiva funcional resulta muy útil en el diagnóstico diferencial entre víctimas $y$ pseudovíctimas. Se incluye sin duda desde el primer momento de la lectura del expediente y durante toda la exploración psicológica, pues los componentes que estamos describiendo se ponen en relación transversal a través del análisis intelectual del psicólogo: pensamos todo el tiempo como psicólogos aplicados a caso único, empleando la herramienta psicológica adecuada en cada momento de la evaluación, y la más completa sería el AFC.

Se precisa una lectura comprensiva muy detallada, que lleve al psicólogo a un análisis exhaustivo para extraer todo el significado de la información y trazar el futuro curso de acción respecto a los objetivos de su investigación psicológica: el planteamiento de la evaluación a partir de la formulación de diversas hipótesis (Muñoz, 2013b; Scott y Manzanero, 2015; Masip y Herrero, 2015b).

Se perfila una herramienta sencilla durante la lectura: plasmar en papel el curso temporal de la acusación en un eje de abscisas (Scott y Manzanero, 2015), su topografía en términos del Análisis Funcional. Contamos a nuestro favor con la forma $a$ priori kantiana que es trascendental a toda percepción y entendimiento: el tiempo, que siempre sigue su curso. Sorprende la cantidad de periciales sobre credibilidad que así se encauzan. 
Vilalta, R. J., Winberg, M. (2016) El análisis funcional de conducta en la evaluación psicológica forense de la credibilidad del testimonio. Behavior \& Law Journal 2 (1), 1-10

La acusación fabricada en casos de abuso sexual infantil intrafamiliar, pretende ocultar los vínculos que la ligan temporalmente a su utilidad y para lograrlo recurre a artificios que confirman por segunda vía su carácter espurio. Especial atención merece aquí, el contexto de revelación original del abuso (una invención necesaria si no lo hubo), que dependiendo de la edad del menor tiene características bastante definidas. Si pedimos a menores y/o adultos que reconstruyan el contexto de revelación, los honestos son contingentes, responden con naturalidad y no se detecta actitud defensiva, coincidiendo además sus versiones. En los testimonios impostados se ofrecen respuestas vagas e inconsistencias, mayor latencia de respuesta, repiten nuestras preguntas, etc., circunstancias que se relacionan con el mayor trabajo cognitivo que necesitan para improvisar y resolver esa dificultad (Porter y Brinke, 2010).

Una madre quedó tan desorientada por la sencilla tarea de relatar el contexto de descubrimiento del abuso a su hijo, que dio explicaciones incongruentes durante más de 8 minutos, sin poder relatar cómo habia descubierto su existencia, emitiendo en cambio diversos mensajes que delataban su impostura.

Resulta de interés también, la contemplación del episodio denunciado dentro de todo el proceso psicológico y relacional que es propio de cada tipología de abuso. Según sean los hechos, tiene que haber un proceso que sustente la existencia del episodio. Si no aparecen estas características contextuales (habitualmente descritas dentro del criterio 19 de CBCA como rasgos característicos de la ofensa) debemos poder explicar razonablemente su ausencia y de no ser así, comprometen la existencia de un episodio aislado. Lo mismo sucede cuando no se incardina con sus antecedentes y consecuentes, algo que también sirve para plantear la entrevista.

Una adolescente denunció a su padre por un episodio puntual de tocamientos, narrado con falta de detalle y un desarrollo poco compatible con: su edad, tipología y evolución. Los abusos los confesó a su hermana universitaria tras un interrogatorio por su mala evolución. La menor acudía desde bacia meses, llevada por su madre, a salud mental por trastornos de conducta, absentismo escolar y malas compañias. La denuncia se produjo pocos días después de que su madre, separada de su padre, le solicitara a éste que fuera a buscarla de madrugada a una fiesta veraniega. Algo que realizó diligentemente, separándola del joven con el que alli alternaba. Para apuntalar su denuncia, afirmaba que la causa de su tratamiento era el acoso paterno, algo que no constaba en los informes de salud mental y contradictorio con la naturaleza puntual del abuso (asi se deducia su falsedad). La denunciante terminó reconociendo ante su madre lo falso del episodio. El AFC situó la denuncia en una tipología de adolescente con trastornos de conducta que así evitaba el control paterno.

\section{SEGUNDO COMPONENTE: PLANTEAMIENTO DE LA EVALUACIÓN}

Todos los hallazgos del estudio del expediente, sirven para plantear la evaluación psicológica, que debe sin duda incluir la tradicional evaluación de la historia de vida y de las características aptitudinales y actitudinales del testigo, pues se necesitan si se llega al Análisis de Validez de la declaración en términos SVA-CBCA, como también dentro de todo el proceso hipotético-deductivo que implica la investigación.

Sabemos que el trabajo de investigación criminal exige la elaboración de guiones sobre lo que supuestamente ocurrió (Ormerod, Barret y Taylor, 2005), un trabajo intelectualmente exigente que puede llevar a errores, entre los que se citan la resistencia cognitiva y el sesgo confirmatorio. Para evitarlos es necesaria una actitud de obstinada racionalidad (Manzanero y González, 2015).

La evaluación psicológica de carácter nomotético resulta imprescindible para barajar las hipótesis basadas en los supuestos de cuantificación y de semejanza (Muñoz, 2013b); pero destacando nuevamente, que la verdadera naturaleza de la investigación de la credibilidad del testimonio es idiográfica. Todo este análisis es de caso único $(\mathrm{n}=1)$. Como en la terapia de conducta, tenemos un sujeto que manifiesta haber sufrido unos hechos con relevancia penal y la investigación se centra en su caso concreto.

Continuar con la evaluación supone tener una actitud estratégica (Masip y Herrero, 2015b), y tras la lectura del expediente podemos planificar qué evaluar -en aplicación de cada técnica posterior- del mejor modo posible para maximizar nuestros resultados. Para ello es necesario este paso de reflexión y 
Vilalta, R. J., Winberg, M. (2016) El análisis funcional de conducta en la evaluación psicológica forense de la credibilidad del testimonio. Behavior \& Law Journal 2 (1), 1-10

planteamiento, manteniéndolo durante toda la evaluación.

Una adolescente habia denunciado agresiones sexuales por dos jóvenes con los que habia alternado una tardenoche festiva. Manifestó en la entrevista que había cosas que habian dicho los denunciados que ella no iba a reproducir, anunciando explícitamente una omisión de información que pudiera ser relevante y que no se atisbaba comprender porqué eludía, suponiendo que se trataba de retazos de sus conversaciones. Preguntada explícitamente por tal omisión, aclaró que se refería a las declaraciones de los imputados: "Es que... llevo unos cuantos días leyendo lo que ellos escribieron, en la declaración...". A la pregunta siguiente del entrevistador: "¿Dices que llevas unos cuantos días leyendo lo que ellos dijeron?", siguió -en tiempo y forma- su gesto de cerrar los párpados echando la cabeza hacia detrás, que expresaba inequivocamente el reconocimiento de un error propio, al revelar tal lectura y la conciencia de la preparación de su testimonio que de ello se deducía y que no se refería a las conversaciones que se suponía habia experimentado.

\section{TERCER COMPONENTE: LA ENTREVISTA}

Lo siguiente es la adecuada obtención del testimonio. Huelga mencionar las características necesarias para la toma de un testimonio válido, que maximice la información relevante y minimice errores de comisión. Nos remitimos a lo que resiste de la entrevista cognitiva (Fisher y Geiselman, 1992), al Protocolo del Acta Michigan de entrevista a menores en contexto forense (Poole y Lamb, 1998) y a la entrevista específica a menores abusados NICHD-2 (Lamb, Orbach, Hershkowitz, Esplin y Horowitz, 2007).

Si la lectura del expediente desvela que existen dudas sobre la originalidad del testimonio infantil por la intervención de profesionales con técnicas inadecuadas, éste debe ser un punto muy relevante, como sucede en las denuncias de abuso inducidas por intervenciones iatrogénicas de profesionales sanitarios.

Una joven de 24 años comenzaba su relato diciendo: "Antes no recordaba cosas que ahora recuerdo,... por la psicóloga". Revelaba después la atormentada joven las prácticas de recuerdo inducido de un episodio de abuso, recreado en consulta en términos de cognición adulta empleando las fotografías de su cuarto cumpleaños, que habian producido tal Falsa Memoria (Loftus,1996).

Se propone una entrevista en la actitud ¿Qué deseas contar? Pero estimulando, sobre todo mediante el silencio del entrevistador, la emisión de un relato episódico analizable. Mediante esta devolución del control al entrevistado se procura alivio y control a la víctima, y se evita toda confrontación con la pseudovíctima. Siempre debe grabarse en vídeo para su posterior estudio, consiguiendo minutos enteros de relato sin apenas intervención del entrevistador.

Encontramos además que aparecen momentos cruciales en la detección de expresiones y emociones significativas durante la entrevista, que es en sí misma todo un proceso a analizar en la dinámica ya señalada de caso único de medidas repetidas. Merecen atención:

La actitud previa y su inicio: sala de espera y actitud de los adultos hacia el menor (alentándole o confortándole); espera impaciente; disposición a relatar directamente; actitud teatral; etc. Frecuentemente se recibe a primeros infantes, supuestamente abusados, que entran llorando amedrentados. Su reacción de temor inicial es instada en la esperanza de impresionar al evaluador. Lo que procura el adecuado diagnóstico, es mantener una actitud estratégica, señalando a su acompañante que así no es posible la evaluación, procurando sacar al menor rápidamente de la consulta y pautando un tiempo fuera. El infante regresa relajado, lo que nos permite evaluar y confirmar además la función inicial.

Una madre con una denuncia fabricada, reconoció que le habia dicho al menor que iban a la consulta del dentista, lo que acreditaba la preparación de su respuesta de temor.

La Eclosión de emociones: llanto forzado durante toda la evaluación o súbito llanto incontenible en el relato del evento central.

El final de la entrevista: satisfacción por la ejecución o fuerte motivación de resultado. En esta parte puede detectarse la ocultación de las instrucciones dadas al menor o del premio que le espera por haber cumplido la tarea:

Una preescolar comunicaba al final de la entrevista que su mamá le habia prometido una muñeca al salir. Este dato aislado no resultaba significativo, pero al entrar su madre a recogerla comenzó a exigirsela insistentemente. 
Vilalta, R. J., Winberg, M. (2016) El análisis funcional de conducta en la evaluación psicológica forense de la credibilidad del testimonio. Behavior \& Law Journal 2 (1), 1-10

\begin{abstract}
Su madre hacia oídos sordos a sus demandas, pretendiendo asi extinguir su reclamo. Su actitud de disimulo confirmaba la función del premio acordado.
\end{abstract}

La entrevista no siempre se detiene con el relato libre, le añadimos función cuando se convierte en estratégica. Narrar un relato ficticio sobre un episodio de agresión complejo es una tarea de alto riesgo, que exige un extraordinario esfuerzo mental de elaboración y puesta en escena. La investigación en mentiras de alto voltaje está en pañales frente a la de las triviales, pues conllevan mucho estrés y trabajo cognitivo y emocional que puede detectar el psicólogo (Porter y Brinke, 2010; Masip y Herrero 2015a). Esfuerzo cognitivo que incluye: recordar el relato; contarlo sin contradicciones intrínsecas ni extrínsecas; monitorizar las reacciones del entrevistador; responder a sus demandas; y ligar toda su conducta verbal y no verbal a las fluctuaciones emocionales propias y contingentes al mismo, ocultando unas y revelando otras.

Sostener este tipo de mentiras exige mayores tareas cognitivas y tensiones emocionales que decir la verdad, lo que da lugar a una sobrecarga cognitiva y emocional que puede detectarse. Este sobresfuerzo procuraría deslices o indicios de engaño que el psicólogo puede identificar. Existen varias investigaciones en esta dirección: Información lingüística (Pennebaker, Francis y Booth,, 2001); Entrevista de conocimiento culpable (Ben-Shakhar y Elaad 2002); Preguntas inesperadas (Vrij, Leal, Granhag, Mann, Fisher, Hillman et al., 2009); Entrevista Abogado del Diablo (Vrij, Fisher, Leal y Mann, 2010); Detección cognitiva de la falsedad (Vrij, Granhag, Mann y Leal, 2011).

Preparar una entrevista estratégica exige un análisis exhaustivo en busca de cuestiones de consistencia dudosa para hacer preguntas inesperadas, que también surgen de la propia dinámica de la entrevista (Masip y Herrero, 2015b). Los psicólogos continuamente incluyen entre sus actividades diagnósticas, revisar sus decisiones en relación (dialéctica) con la dinámica de la situación de entrevista (Groenier, Beerthuis, Pieters, Witteman y Swinkels, 2011). La entrevista forense debe ser dinámica y está en continua revisión sobre su objeto y dirección. Podemos utilizar descansos en la entrevista para analizar lo obtenido, planificar y seguir después.

\section{CUARTO COMPONENTE: LA VALIDEZ Y CALIDAD NARRATIVA DEL TESTIMONIO}

El citado análisis CBCA-SVA, pretende la acreditación del relato a través de la comprobación de su calidad narrativa, entendiendo válida la hipótesis de Undeutsch (1989), según la cual se puede distinguir el relato real del inventado en virtud de sus características (Amado, Arce y Fariña, 2015). Es la técnica que más se conoce y aplica y en la que menos nos detendremos. Los mismos Arce y Fariña (2005) proponen su Sistema de Valoración Global de la credibilidad del testimonio (Arce y Fariña, 2005) que ha tenido bastante uso y que algunos aplican como un protocolo. Toda la investigación sobre credibilidad de este análisis está centrada en la calidad y validez del testimonio de abuso sexual, y se puede complementar con la consistencia de sus secuelas psicológicas.

El problema del análisis de la calidad narrativa estriba en los factores que pueden limitarla: la edad del menor, su competencia lingǘstica, la sencillez o complejidad del episodio, o una posible actitud defensiva que limite sus verbalizaciones. Resulta además aplicable únicamente a menores sin experiencia sexual, y como señalan Manzanero y González (2015), los criterios de contenido por sí mismos no parecen todo lo útiles que se esperaba para distinguir relatos reales y falsos y dependen mucho de los conocimientos y experiencia del profesional, algo que parece justificar el enfoque idiográfico, deductivo y funcional que proponemos para la evaluación psicológica del testimonio.

\section{QUINTO COMPONENTE: EVALUAR EL ENGAÑO}

\footnotetext{
El contrapunto al SVA-CBCA lo encontramos en la detección del engaño y las características propias de la mentira. Ambos enfoques son compatibles y pueden evaluarse simultáneamente. De hecho, el análisis de la validez del testimonio mediante el SVA pretende un contraste de hipótesis y detectar inconsistencias, que se pretender completar añadiendo todo el desarrollo metodológico ligado a la detección de la simulación y del engaño.
} 
Vilalta, R. J., Winberg, M. (2016) El análisis funcional de conducta en la evaluación psicológica forense de la credibilidad del testimonio. Behavior \& Law Journal 2 (1), 1-10

Las investigaciones que pretenden encontrar un indicador universal del engaño acaban despreciando la posibilidad de su detección mediante indicadores fisiológicos y lenguaje verbal y no verbal (Masip, 2005). Pero en estas mentiras de alto riesgo y con este método estratégico, aumentamos la posibilidad hacerlo mediante conocimiento del contexto y estrategia, pues existe un cuerpo de conocimiento que sugiere que así las pistas verbales y no verbales del engaño se vuelven más evidentes ( Bond, Levine y Hartwig, 2015; Masip y Herrero 2015a; Porter y Brinke, 2010; Vrij, 2015b;). Podemos encontrar diversos indicios del engaño en determinados usos lingüísticos, en la comunicación no verbal, en la simulación de secuelas psicológicas y sobre todo en su funcionalidad.

Son útiles también, los conocimientos que se derivan del diagnóstico diferencial en contextos propios de simulación y fingimiento. La pseudovíctima realiza un trabajo psicológico muy similar al de quien simula un trastorno.

Entre los instrumentos más populares para la detección del engaño están los derivados de la valoración del lenguaje no-verbal que acompaña al discurso: el estudio de las expresiones $y$ microexpresiones, deslices verbales o gestuales, que escapan al control del sujeto y que es posible atribuir a determinada emoción, como pretende el Protocolo FACS de Ekman y Friesen (1978). No es propiamente dicho análisis el que se propone utilizar sistemáticamente, pero en ocasiones resulta relevante.

Una joven relataba una compleja noche con el que habia sido su novio durante los últimos años. Tras romper ese mismo día por una infidelidad de ella, decidieron pernoctar juntos en el domicilio del joven, en el que se encontraban su madre y su hermano adolescente. El relato de la joven incluia su retención en la habitación, agresiones, estrangulamientos y tres violaciones. Después de una hora de relato en constante y forzado llanto, se le preguntó si tal relato era lo que habia sucedido aquella noche. Respondio: "Si, sí, si." mientras que con la cabeza hacía otros tantos gestos de negación.

Pese a que en ocasiones como la descrita sea tan relevante tal desliz emblemático, en realidad se trata de detectar expresiones emocionales genuinas durante el relato, y entender funcionalmente cómo van asociadas al relato honesto o falso. Ekman (2001) destacaba tres emociones claramente diferenciables en el gesto de los testimonios de menores: miedo, satisfacción y vergüenza o culpa.

En una expresión de miedo, el objetivo sería diferenciar entre el miedo a la revelación del episodio de abuso o sus consecuencias, y el miedo a las consecuencias de su falsedad, veamos lo segundo:

Tras una primera parte en la que un niño hablaba con afecto y naturalidad sobre su padre, cumplió la tarea encomendada realizando el relato rocambolesco de unos tocamientos por parte del mismo en presencia y al dictado de su abuela paterna; sin embargo tal relato no le resultaba agradable $y$ mostraba un gesto de contención del llanto que escondia al evaluador. Tal tenaz ocultación funcional de su emoción resulta impropia de un testimonio bonesto. Finalmente comunicó el temor que le provocaba su madre, quien le habia advertido que si no contaba los tocamientos ella terminaría en la cárcel por mentir.

En el gesto de satisfacción debemos distinguir entre el alivio por liberarse del secreto y la diversión por la ejecución del engaño, por lo increíble de lo referido o por la venganza pretendida.

La menor de 10 años con amnesia fingida antes descrita, no pudo disimular su risa cuando pretendia ignorar la fecha de su cumpleaños. Lo que se confirmaba en segunda vía cuando intentaba ocultarla rápidamente.

En la vergüenza o culpa distinguiremos entre la provocada por la naturaleza repulsiva de los hechos o por delatar al abusador, y la que suscita la mentira. La dificultad en todos los casos, estriba en asociar la expresión a su emoción correcta y atribuirla inequívocamente a la motivación subyacente que la suscita, realizando un atinado AFC.

Otra perspectiva en la detección del engaño se centra en el análisis estrictamente lingüístico del testimonio. En el ámbito policial es popular el discutido Scientific Content Analisys (SCAN) de Sapir (1998), que plantea que todo el mundo desea informar de los hechos traumáticos y solicita un relato libre de lo sucedido. Alerta sobre los relatos cortos o 
demasiado ordenados, o de si no existe un equilibrio global de la declaración en sus 3 partes de planteamiento-nudo-desenlace. La técnica destaca las pistas que ofrece el uso: de los distintos tiempos verbales; de los pronombres de $1^{a}$ persona; del singular o plural en las acciones (que podría denotar implicación en lo sucedido); del artículo determinado e indeterminado (el/un); de posesivos; de nexos temporales innecesarios en el discurso "un poco después", cortinas que esconden acciones intermedias que no se exponen; de las locuciones que indican falta de implicación en detalles críticos (creo recordar que...); o de la demora en responder a preguntas clave. Nuevamente lo valioso es observar estos indicadores y comprobar si son relevantes o no en la cadena conductual del AFC.

Una adolescente de 13 años reveló unos supuestos abusos por parte de su padre con una nota manuscrita entregada en su instituto, que iniciaba: "Hace más o menos un trimestre..." Llamó la atención el uso del vocablo "trimestre" para datarlos, pues es término ligado al ámbito escolar y no a experiencias de abuso incestuoso externas al mismo. Lo cierto es que tal pista revelaba la función de la falsa denuncia, pues al día siguiente su madrastra iba a visitar a la tutora y la chica habia dado un giro negativo en su conducta y escolaridad.

Efectivamente, parece más eficaz tratar de detectar el engaño y la peculiar conducta de acusar falsamente de abuso sexual, que acreditarlo a través de la calidad del relato. En uno de los pocos estudios de campo empleando el SCAN (Driscoll, 1994), un experto clasificó correctamente el $73 \%$ de los testimonios auténticos antes de pasar por el polígrafo, y fue capaz de clasificar correctamente el $95 \%$ de los falsos. Nuestra evaluación pretende esa especificidad, que permita descartar de forma sistemática el engaño consiguiendo: "... informar de las hipótesis que no son correctas pues van en contra de la evidencia cientifica." (Scott y Manzanero, 2015).

El vídeo es una herramienta imprescindible en la evaluación del testimonio, permite la observación del gesto y el estudio minucioso de las verbalizaciones, además facilita enormemente su análisis funcional.

\section{EL COMPONENTE INTEGRAL: EL ANÁLISIS FUNCIONAL DE CONDUCTA}

Los elementos de juicio para una evaluación psicológico-forense son las hipótesis y las deducciones de un proceso lógico-deductivo. Aunque el contexto de evaluación sea el forense, la filosofía de esta perspectiva es rigurosamente psicológica: aplicar la técnica del AFC al estudio de la credibilidad. Evidentemente, este enfoque se apoya en los desarrollos metodológicos de las evaluaciones centradas en buscar criterios de calidad en los testimonios de abuso sexual y en los indicadores del engaño, pero otorga valor primordial al enlace lógico de las evidencias de toda la valoración efectuada.

En el estudio individual de cada caso, acaba resultando poco importante si algunos criterios puntuales suelen asociarse a acusaciones falsas o a genuinas, pues cada caso reúne peculiaridades únicas que -según cómo se articule con el resto- concluirán que una determinada expresión o conducta, por su función, sea criterio de credibilidad en un caso y de desconfianza en otro, pues los comportamientos tienen funciones diferentes en la vida de los sujetos según sus circunstancias y motivaciones, solventándose así la paradoja señalada por Vrij (2015) de que algunos criterios aparecen como indicadores de credibilidad en CBCA y de engaño en SCAN.

La diferencia está en detectar, para cada caso, si la conducta de acusar cumple una función psicológica en lugar de corresponderse ésta con la disfunción o disposición que sería esperable de una experiencia real de abuso. El análisis debe ser parsimonioso y exhaustivo. Para la comprensión de un problema real se precisa integrar toda la información. Describir cómo se relacionan estímulos y respuestas, las características particulares del sujeto, cómo actúan sus cogniciones sobre cuestiones cruciales de su comportamiento, y cómo las variables del contexto influyen en todo ello. Se exige tiempo para el análisis, para la reflexión y la redacción de su lógica en un poderoso esquema general del evaluado (Haynes, 1990).

Las diferentes catas del análisis psicológico se realizan en series $\mathrm{AB} A B$, ligando los antecedentes y los comportamientos examinados de diferentes momentos: episodios de abuso, periodo de 
Vilalta, R. J., Winberg, M. (2016) El análisis funcional de conducta en la evaluación psicológica forense de la credibilidad del testimonio. Behavior \& Law Journal 2 (1), 1-10

ocultamiento, episodio de revelación, denuncia policial, declaraciones judiciales, informes previos y la entrevista forense. Una vez analizados esos episodios de la vida del evaluado, se enlazan en la línea cronológica de la evolución de los hechos, sus comportamientos, creencias y actitudes. Esas series temporales son analizadas en cuanto a la consistencia lógica de su evolución.

La gran ventaja del AFC sería su especificidad, algo de lo que adolece aisladamente la CBCA, que puede causar un elevado porcentaje de falsos positivos, pues -pese a la SVA- padece en su aplicación de cierto sesgo confirmatorio, al no contemplar más detalladamente todavía hipótesis alternativas a la del abuso. Nuestra propuesta podría mejorar muy considerablemente la detección de denuncias directamente falsas, lo que en una perspectiva de caso único reduciría esos falsos positivos. Difícilmente puede cometerse un error tipo I cuando se detecta un engaño y además se acredita la función psicológica del mismo.

Es decir, $\underline{\text { la especificidad del análisis funcional sería }}$ máxima al describirse para cada caso concreto cómo el compendio de evidencias contrastadas y las pistas verbales (testimonio, análisis lingüístico), no-verbales y contextuales se articulan en hipótesis consistentes que convergen y explican, en forma de argumentos, el porqué de la falsedad o lo infundado de esa acusación concreta.

Si no encontramos engaño, hallamos criterios de validez y credibilidad del testimonio y el Análisis Funcional no revela motivaciones espurias, sino la

\section{REFERENCIAS}

Amado, B., Arce, R. y Fariña, F. (2015). Undeutsch hypothesis and Criteria Based Content Analysis: A meta-analytic review. The European Journal of Psychology Applied to Legal Context 7, 312

Arce, R. y Fariña, F. (2005). Peritación psicológica de la credibilidad del testimonio, la huella psíquica y la simulación: el sistema de evaluación global (SEG). Papeles del psicólogo, 26(92), 59-77.

Ben-Shakhar, G. y Elaad, E. (2002). Effects of questions repetition and variation on the efficiency of the Guilty Knowledge Test: a reexamination. Journal of Applied Psychology 87, 972-977.

Bond, C. F., Levine, T. R. y Hartwig, M. (2015). New Findings in Non-Verbal Lie Detection. En P.A. Granhag, A. Vrij y B. Verschuere (Ed) Detecting Deception: Current Challenges and Cognitive Approaches (37-58). Chichester: Wiley. evolución propia del sujeto -en términos cognitivos, emocionales y conductuales- esperable de tal evento, entonces podemos estar en condiciones de proponer su credibilidad.

Al final, la evaluación del abuso genuino nos remite a: la disfuncionalidad de tales conductas sexuales, a la aparición de indicadores específicos e inespecíficos del abuso, al Síndrome de acomodación al abuso (Summit, 1983) o a las tipologías de abusadores sexuales (intrafamiliares/ conocidos/ desconocidos, seductores/ merodeadores/ asaltadores / cibernéticos, etc.).

Por el contrario, si la evaluación nos remite a una funcionalidad de la denuncia y su análisis adquiere sentido considerando el engaño, la evaluación remite a diferentes tipologías de denuncias infundadas o falsas. Éstas incluyen: los contextos de interferencias parentales en las visitas; los trastornos delirantes; las falsas memorias por mala praxis o a cargo de familiares o conocidos más o menos honestos; los adolescentes manipulando el sistema en contextos de protección (Mikkelsen, Guthiel y Emens, 1.992); las inversiones de rol para ocultar infracciones o tener coartadas; o la necesidad de atenciones emocionales (Kanin, 1994).

Seguimos la recomendación de Vrij, Akehurst, Soukara y Bull (2004) pues combinamos CBCA-SVA con procedimientos de evaluación del contenido verbal, con indicadores conductuales del engaño y añadimos además un componente más valioso y que se ajusta como un traje de alta costura a la medida de cada caso: el Análisis Funcional de Conducta.
Driscoll, L. (1994). A Validity Assessment of Written Statements from Suspects in Criminal Investigations Using the SCAN Technique. Police Studies, 17, 77-88.

Ekman, P. y Friesen, W. (1978). Facial Action Coding System: A Technique for the Measurement of Facial Movement. Consulting Psychologists Press: Palo Alto.

Ekman, P. (2001). Telling Lies. Berkley Books: NewYork.

Fisher, R. y Geiselman, R. (1992). Memory-enhancing Techniques for Investigative Interviewing: The Cognitive Interview. Charles C. Thomas, Publisher.

Groenier, M., Beerthuis, V. R., Pieters, J. M., Witteman, C. L., y Swinkels, J. A. (2011). Psychologists' diagnostic processes during a diagnostic interview. Psychology, 2(09), 917.

Haynes S.N. y O`Brien, H. (1990). Functional Analysis in behavior therapy. Clinical Psychology Review.10, (6). 649-668. 
Vilalta, R. J., Winberg, M. (2016) El análisis funcional de conducta en la evaluación psicológica forense de la credibilidad del testimonio. Behavior \& Law Journal 2 (1), 1-10

Kanin, E. (1994). False rape allegations. Archives of Sexual Behavior. 23(1), 81-92.

Lamb, M., Orbach Y., Hershkowitz, I.; Esplin P. y Horowitz D. (2007). NICHD 2. A structured forensic interview protocol improves the quality and informativeness of investigative interviews with children: A review of research using the NICHD Investigative Interview Protocol. Child Abuse and Neglect, 31 (11), 1201-1231.

Loftus, E. (1996). The Myth of Repressed Memory: False Memories and Allegations of Sexual Abuse. Macmillan.

Manzanero, A. L. (2001). Procedimientos de evaluación de la credibilidad de las declaraciones de menores víctimas de agresiones sexuales. Psicopatología clínica, legal y forense, 1(2), 51-71.

Manzanero, A. L., y González, J. L. (2015). Modelo holístico de evaluación de la prueba testifical (HELPT). Papeles del Psicólogo, 36(2), 125-138.

Masip, J. (2005). ¿Se pilla antes a un mentiroso que a un cojo? Sabiduría popular frente a conocimiento científico sobre la detección no-verbal del engaño. Papeles del Psicólogo, 25, 7891.

Masip, J., y Herrero, C. (2015a). Nuevas aproximaciones en detección de mentiras I. Antecedentes y marco teórico. Papeles del Psicólogo, 36(2), 83-95.

Masip, J., y Herrero, C. (2015b). Nuevas aproximaciones en detección de mentiras II. Estrategias activas de entrevista e información contextual. Papeles del Psicólogo, 36(2), 96-108.

Mikkelsen, E., Guthiel, T. y Emens, M. (1.992). False abuse alegations by childrens and adolescents: Contextual factors and clinical subtypes. American Journal of Psychotherapy, 55, 556-570.

Muñoz, J. M. (2013a). La evaluación psicológica forense del daño psíquico: propuesta de un protocolo de actuación pericial. Anuario de Psicología Jurídica, 23, 61-69.

Muñoz, J. M. (2013b). El proceso de evaluación psicológica forense: diferencias con la evaluación clínica. En Manual Docente de Psicología Clínica, Legal y Forense I, 213-230.

Ormerod, T. C., Barret, E. C. y Taylor, P. J. (2005) Investigative sense making in criminal contexts. En J.M.C. Schaagen (Ed.), Proceedings of the Seventh International NDM Conference, 1-12, Amsterdam june 2005

Pennebaker, J.W., Francis, M.E.; Booth, R.J. (2001). Linguistic Inquiry and Word Count: LIWC. Mahwah, NJ: Erlbaum Publishers.

Poole, D. A., y Lamb, M. E. (1998).Investigative interviews of children: A guide for helping professionals. American Psychological Association.

Porter, S. y Brinke, L. (2010). The truth about lies: What works in detecting high-stakes deception? Legal and Criminological Psychology, 15, 57-75.

Sapir, A. (1998). L.S.I. Advanced Workshop on Scientific Content Analysis. Phoenix: Laboratory for Scientific Interrogation.

Scott, M. T. y Manzanero, A. L. (2015). Análisis del expediente judicial: Evaluación de la validez de la prueba testifical. Papeles del Psicólogo, 36(2), 139-144.

Steller, M. y Köhnken, G. (1989): Criteria-Based Statement Analysis. Credibility Assessment of Childrens Testimonies in Sexual Abuse Cases. En D.C. Raskin (Ed.), Psychological Methods for Investigation and Evidence (217-245). New York: Springer.
Summit, R. C. (1983). The child sexual abuse accomodation syndrome. Child abuse \& neglect, 7(2), 177-193.

Trankell, A. (1972). Reliability of Evidence: methods for analyzing and assessing witness statements. Stockholm: Beckman (Orig.1963)

Undeutsch, U. (1989). The development of statement reality analysis. En J.C. Yuille (Ed.), Credibility assessment (pp. 101121). Dordrecht, Países Bajos: Kluwer.

Vrij, A., Akehurst, L., Soukara, S., \& Bull, R. (2004). Detecting deceit via analyses of verbal and nonverbal behavior in children and adults. Human communication research, 30(1), 841.

Vrij, A., Leal, S., Granhag, P. A., Mann, S., Fisher, R. P., Hillman, J., y Sperry, K. (2009). Outsmarting the liars: The benefit of asking unanticipated questions. Law and buman behavior, 33(2), 159-166.

Vrij, A.; Fisher, R.P.; Leal, S.; Mann, S. (2010). Detecting true and false opinions: The Devil's Advocate approach as a lie detection aid. Acta Psychologica, 134(3), 323-329.

Vrij, A.; Granhag, P.A.; Mann, S.; Leal, S. (2011). Outsmarting the Liars: Toward a Cognitive Lie Detection Approach. Psychological Science 20, 28-32.

Vrij, A. (2015). Verbal Lie Detection Tools: Statement Validity Analysis, Reality Monitoring and Scientific Content Analysis. En P.A. Granhag, A. Vrij y B. Verschuere (Ed) Detecting Deception: Current Challenges and Cognitive Approaches (3-35). Chichester: Wiley.

Vrij, A. (2015b). A cognitive approach to lie detection: Statement Validity Analysis, Reality Monitoring and Scientific Content Analysis. En P.A. Granhag, A. Vrij y B. Verschuere (Ed) Detecting Deception: Current Challenges and Cognitive Approaches (205-229). Chichester: Wiley. 